\title{
Neurocysticercosis and epilepsy in developing countries
}

\author{
Deb K Pal, Arturo Carpio, Josemir W A S Sander
}

Neurosciences Unit, Institute of Child Health, University College London, UK $\mathrm{D} \mathrm{K} \mathrm{Pal}$

Faculty of Medicine and Research Institute, University of Cuenca, Cuenca, Ecuador A Carpio

Epilepsy Research Group, Institute of Neurology, 33 Queen Square, University College London, UK J W A S Sander

Correspondence to: Dr DK Pal, Neurosciences Unit, Institute of Child Health, University College London, The Wolfson Centre, Mecklenburgh Square, London WC1N 2AP, UK

email d.pal@ucl.ac.uk

Received 6 August 1999 and in revised form 6 October 1999

Accepted 28 October 1999

\begin{abstract}
Neurocysticercosis is a disease of poverty and underdevelopment. Little is known about the natural history of the infection in humans, but some of the mechanisms whereby the parasite remains silent and evades the host immune response are understood. Symptomatic neurocysticercosis usually results from host inflammatory response after parasite death, and the clinical manifestations can be diverse. There is no evidence that cysticidal treatment does more good than harm in addition to conventional antiepileptic treatment. Population control measures involving immunisation or mass treatment have not shown long term effectiveness.
\end{abstract}

Epilepsy, similarly to neurocysticercosis, is a largely unrecognised but increasing burden on the welfare and economies of developing countries. The technology of drug treatment and psychosocial rehabilitation is well known but requires widespread and effective dissemination at low cost. There is little epidemiological data on risk factors for epilepsy in developing countries on which to base prevention strategies. The public health prioritisation of chronic disorders such as epilepsy remains a challenge for policy and practice in developing countries.

For both neurocysticercosis and epilepsy, there is a dilemma about whether limited public resources would better be spent on general economic development, which would be expected to have a broad impact on the health and welfare of communities, or on specific programmes to help individual affected people with neurocysticercosis and epilepsy. Either approach requires detailed economic evaluation. (F Neurol Neurosurg Psychiatry 2000;68:137-143)

Keywords: neurocysticercosis; epilepsy; developing countries; service delivery

The immense burden of epilepsy is a growing problem in developing countries where the incidence of epilepsy may be higher than in western countries. ${ }^{1}$ Three quarters of the 50 million people with epilepsy live in the poor countries of the world and up to $94 \%$ are untreated. ${ }^{2}{ }^{3}$ Extending appropriate services to these people will be one of the great challenges of the new millenium. Unfortunately little is known about the causes of epilepsy in developing countries. However, many studies from Latin America have shown that infection of the brain by the larvae of the pork tapeworm Taenia solium is an important cause of epileptic seizures in endemic communities. ${ }^{4-6}$ Many recent reviews have covered in detail the pathology, ${ }^{7}$ parasitology, ${ }^{8}$ clinical features, ${ }^{6}$ and diagnostic criteria $^{9}$ of neurocysticercosis. This review concentrates on recent developments in the immunopathogenesis of neurocysticercosis, the controversy over clinical classification, and the evidence base for different treatment approaches. The challenges for the population control of cysticercosis and epilepsy in the developing world are summarised.

\section{Neurocysticercosis}

EPIDEMIOLOGY

Taenia solium is endemic in Latin America, India, and China, and may also be endemic in sub-Saharan Africa, although there are few studies. ${ }^{10}{ }^{11}$ Poor hygiene and living conditions, allowing pigs access to human faeces, put people at risk of developing cysticercosis. ${ }^{42-15}$ In endemic countries, the disease is also widely prevalent in urban, middle class areas. ${ }^{16}$ Migration from the countryside and the rise of urban slums obviously influence the changing epidemiology of cysticercosis. $T$ solium infections have also been imported by migrant workers into the United States. ${ }^{17}$

Neurocysticercosis is of great economic relevance, resulting from the cost of medical treatment, lost working days, and losses due to livestock condemnation. A minimum estimate of the cost of admissions to hospital and wage loss for neurocysticercosis in the United States (a non-endemic country) was $\$ 8.8$ million annually, wheres estimated treatment costs in Mexico were $\$ 89$ million, and Brazil $\$ 85$ million. ${ }^{18}$

\section{PATHOLOGY}

Life cycle biology

In the first stage, the human host ingests diseased (measly) pork containing viable cysticerci, from within which the scolex of the metacestode evaginates in the gut and attaches to the intestinal mucosa. ${ }^{8}$ The tapeworm 
matures over 2-3 months to achieve a length of 2-7 m. Gravid segments may contain 50-60 000 eggs which are passively released in small groups in faeces, two or three times a week, often unknown to the host. After ingestion, eggs hatch and activate in the pig small intestine and develop in striated muscle and the CNS. When humans accidentally become intermediate hosts by ingesting eggs, the life cycle is completed in a similar way in muscle, skin, and CNS.

\section{Human pathology}

Our understanding of natural human infection depends on studies of expatriates from endemic zones, and postmortem data. Initially, there is an asymptomatic period after egg ingestion lasting many years or even for life. The time between infection and symptoms in neurocysticercosis depends on number, size, type, condition, and site of cysts in the brain. A study of British soldiers with neurocysticercosis returning from India showed that most developed seizures $2-8$ years after infection. ${ }^{19}$ Postmortem studies in endemic zones show that about $80 \%$ of infections are asymptomatic. ${ }^{20}$ However, the risk of intracranial infection after $T$ solium egg ingestion is unknown.

\section{IMMUNOLOGY AND PATHOGENESIS}

Evidence from animal models and clinical studies shows that cysticerci remain clinically silent as a result of active immune tolerance, and that symptomatic parenchymal disease occurs at the time of larval degeneration or death by cysticidal therapy. Human neurocysticercosis treatment studies show rises in IgG, interleukin-2, and neopterin in the $\mathrm{CSF}^{21} \mathrm{~A}$ study of patients with hepatic echinococcosis (another human cestodiasis) suggests that a switch in IgG subclass response from IgG1 to IgG4 might occur as the disease progresses from its asymptomatic to symptomatic stages. ${ }^{22}$ Recently it has been reported that eotaxin and interleukin-5 concentrations are raised in the serum of patients with symptomatic neurocysticercosis, and interleukin-5 and interleukin-6 concentrations are also raised in the CSF, possibly indicative of an acute phase response. ${ }^{23}$

Epidemiological and clinical findings suggest that individual immunological responses to cysticercosis might have a genetic basis. Firstly, Guatemalan population studies have shown no association between $T$ solium seropositivity and epileptic seizures in a highly endemic area. ${ }^{12}$ This could be explained by differences in population genetics or parasite strains, although there is little evidence of the second. ${ }^{8}$

Secondly, seizures are more common with multiple lesions, ${ }^{24}$ and leucocyte chemotaxis is impaired in patients with multiple neurocysticercosis lesions. ${ }^{25}$ Multiple lesions are much less common in India than in Latin American countries. ${ }^{26}$ Preliminary association of epilepsy in neurocysticercosis with HLA type I has been reported in India. ${ }^{27}$ HLA-DR polymorphisms have been demonstrated in various infections including leishmaniasis, onchocerciasis, filariasis, hepatitis, and malaria, whereas polymor- phisms of tumour necrosis factor $\alpha$ and of the membrane protein ICAM-1 are associated with increased risk of death from cerebral malaria. ${ }^{28-30}$ It is hypothesised that HLA differences might also determine the risk of intracranial infection or symptomatic parenchymal disease in neurocysticercosis.

\section{CLINICAL PRESENTATION}

There are wide variations of clinical manifestations of neurocysticercosis. These are a consequence of inflammation around a cyst(s), space occupation and impedance to the flow of CSF, less commonly meningeal or vascular inflammation, and non-CNS disease. Seizures are the most common symptom in $70 \%-90 \%$ of patients. ${ }^{6926}$ These may occur both when a cyst is degenerating, ${ }^{31}$ or around a chronic, calcified lesion. $^{32}$

Electroencephalography shows focal or generalised abnormalities, or no abnormality in neurocysticercosis epilepsy. Examination of CSF in neurocysticercosis usually yields mild abnormalities such as increased protein or pleocytosis, not always eosinophils. Interestingly, the proportion of seizures reported as generalised tonic-clonic ranges from $28 \%$ to $68 \%,{ }^{53}{ }^{34}$ despite the presence of a focal lesion.

Some $10 \%-20 \%$ of patients present with ventricular cysts, sometimes also with seizures or with meningeal inflammation. Symptoms include nausea, vomiting, headache, ataxia, and confusion. Focal neurological deficits are uncommon. Patients with cysts in the basal cisterns can present with meningeal signs, hydrocephalus, vasculitis, and stroke. ${ }^{9}$ Rarer neurological manifestations have also been reported-namely, altered mental state; spinal cysticercosis with radicular pain or paraesthesiae, or progressive cord compression; ophthalmic cysticercosis; migraine headaches; and neurocognitive deficits. ${ }^{9}$ Cysticercal encephalitis, with multiple parenchymal cysts, an associated inflammatory response, and diffuse cerebral oedema is a rare presentation, often in young girls; these patients are at risk of severe neurological sequelae. Intracranial hypertension and meningeal neurocysticercosis are uncommon in India. ${ }^{35}$ Subcutaneous cysticercosis is much more common in China than in Latin America or India.

\section{Single enhancing CT lesions}

Solitary enhancing CT lesions have commonly been described in India. ${ }^{36}$ It is still unknown why single lesions are a more common presentation in India than multiple lesions. These single lesions are seen as areas of increased signal on MRI, and are mostly attributed to neurocysticercosis on the basis of resolution or calcification over months with conservative treatment (antiepileptic drugs). Tuberculosis is the primary differential diagnosis, but pyogenic abscess, fungal infection, vasculitis, and neoplasms can account for similar appearances. ${ }^{7}$ Criteria for differentiating cysticerci and solitary tuberculosis lesions have been proposed by Rajshekar et $a .^{38}$ In their histologically established series, intracranial hypertension and progressive neurological deficit were not seen 
with neurocysticercosis; all neurocysticercosis lesions were less than $20 \mathrm{~mm}$ in size, mostly regular in outline, and not associated with midline shift.

Diagnosis

Neuroimaging is essential to the diagnosis of neurocysticercosis (see fig 1 and fig 2) Brain MRI is superior for showing intraventricular or subarachnoid cysts, and for showing inflammation around a cyst, ${ }^{39}$ whereas CT is better for showing the calcification of inactive lesions. There may be single or multiple cysts in different pathological stages. Carpio has proposed a classification system that corresponds to the viability of the parasite: active, transitional, and inactive. ${ }^{7}$ In the active stage, the cyst is asymp- tomatic, and on CT appears as a rounded, hypodense area, or with CSF-like signal on MRI. Both MRI and CT can show the presence of an eccentric mural nodule (the invaginated scolex), an appearance, when multiple, which is pathognomic of neurocysticercosis (starry night effect). As the cyst degenerates, it goes through a transitional stage, with diffuse hypodense appearance and irregular border on CT, enhancing with contrast. On MRI T2 images, these show as low signal areas. Finally when the cyst dies, it may disappear or end up as an inactive calcified nodule of homogenous high density on CT, or low intensity on proton weighted MRI.

Standard enzyme linked immunosorbent assay (ELISA) techniques have disappointing

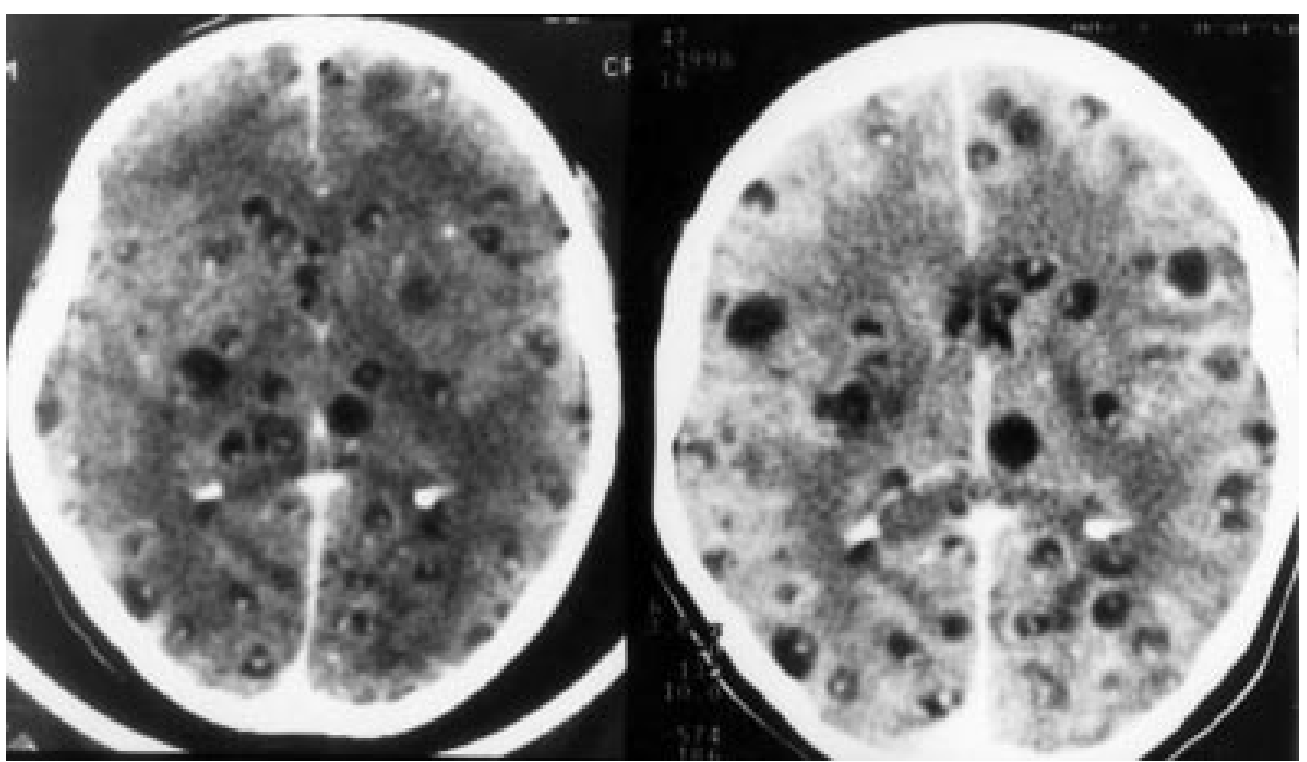

Figure 1 Postcontrast CT of a patient with seizures and intracranial hypertension syndrome, who received albendazole treatment. Left: multiple active cysts with the scolex in their interior (vesicular phase) and calcifications. Right: 16 months after treatment more cysts appeared, and some of them increased in size.

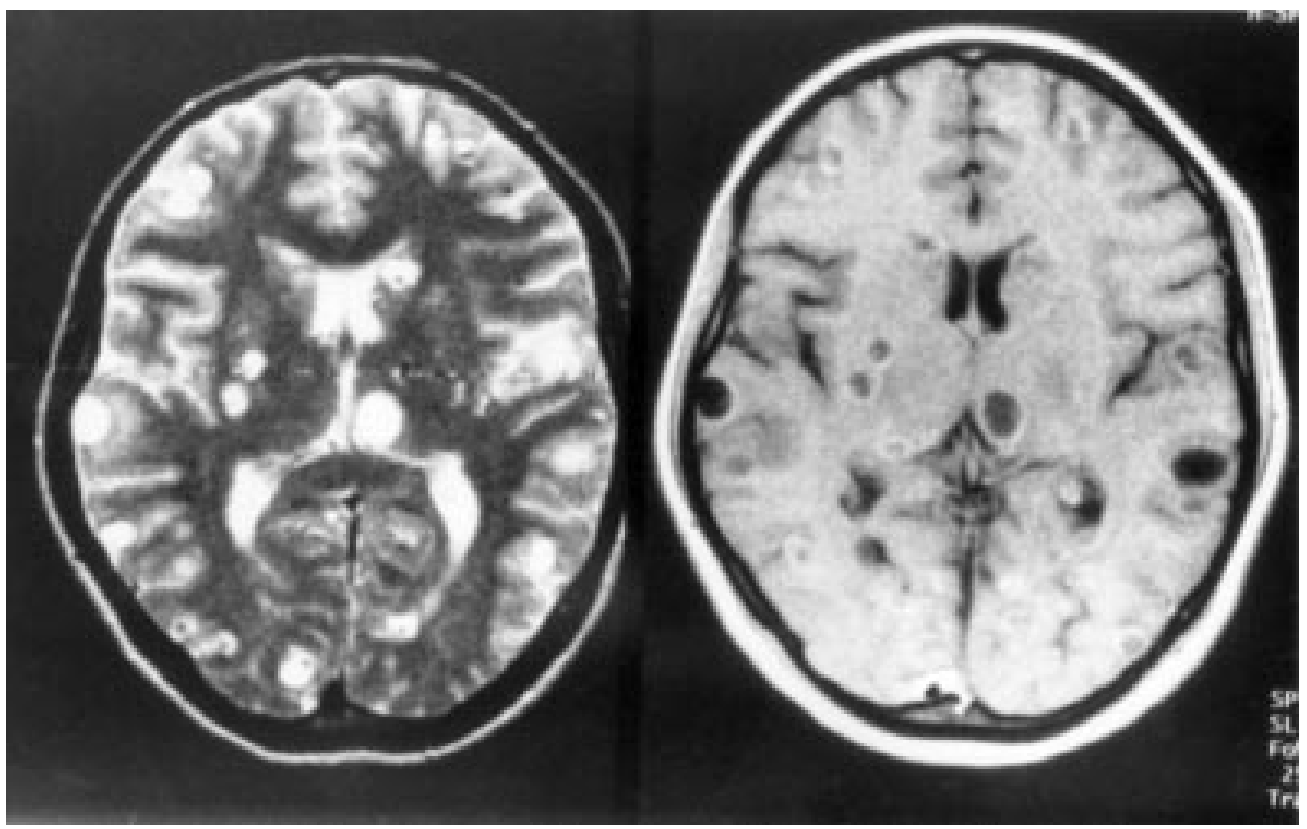

Figure 2 Brain MRI of the same patient of fig 1 (7 months after last CT), who received additional treatment using praziquantel. Left: T2 weighted MRI shows multiple cysts. Right: gadolinium enhanced T1 weighted MRI shows cortical active cysts on parietal lobes and multiple transitional cysts disseminated in both hemispheres. 
sensitivity and specificity in routine clinical or epidemiological use. ${ }^{40}{ }^{41}$ False negative serology can result because of immune tolerance, inactive disease, or localised antibody production in the CSF. False positive serology can result from past infection with $T$ solium or cross reactivity with other helminth species. Newer enzyme linked immunoelectrotransfer blot (EITB) assays on serum or CSF have much higher claimed sensitivity (95\%) and specificity $(100 \%)^{42}$ in Latin American samples.

However, EITB test properties are less good for solitary enhancing CT lesions in India. ${ }^{43}$ In Ecuador, more than $50 \%$ of patients with neurocysticercosis diagnosed by $\mathrm{CT}$ were negative by EITB test; conversely, $18 \%$ with positive EITB had neurocysticercosis parenchymal lesions on $\mathrm{CT}^{16}$ indicating that EITB and neuroimaging should be used in conjunction to increase diagnostic sensitivity. Recently, an antigen detection assay specific for viable metacestodes in CSF has been created. $^{44}$ Immunodiagnostic kits are unfortunately difficult to obtain in endemic countries, so the use of the EITB and other special assays may only be restricted to research studies.

Del Brutto et al have proposed international diagnostic criteria for neurocysticercosis using a combination of clinical, radiological, serological, and epidemiological factors. ${ }^{45}$ These have been criticised for complexity and difficulties in clinical and epidemiological application, and a satisfactory consensus is yet to be reached. ${ }^{46}$

MANAGEMENT: CASE AND COMMUNITY

Clinical case management

The presence of viable parenchymal cysts is not usually associated with symptoms. Most patients with neurocysticercosis present with seizures and in most cases (75\%), these are easily controlled with antiepileptic monotherapy with eventual remission. The natural history of these lesions is for resolution within 2 years. ${ }^{24} 47$ Clinical controversy has centred around the role of cysticidal agents and steroids for epilepsy associated with symptomatic neurocysticercosis. Cysticidal agents in current use for neurocysticercosis include praziquantel and albendazole. Praziquantel has the disadvantage that its hepatic metabolism is inducable by phenobarbital and phenytoin. Cysticidal therapy seems to hasten radiological resolution of cysts but can be associated with an exacerbation of neurological symptoms and there is also the possibility of massive cerebral oedema and death in some patients who have multiple cysts. ${ }^{48}$ Some authors have advocated simultaneous administration of steroids to reduce the inflammatory response and exacerbation of symptoms, but the safety of this has not been evaluated. There have been claims that more patients remain seizure free after cysticidal treatment. ${ }^{32}$ Randomised clinical trials of cysticidal therapy versus placebo for neurocysticercosis have not shown any clinical benefit of cysticidal therapy. ${ }^{49}$ A possible increase in risk of hydrocephalus, and increased seizures during treatment, was also identified in the treated group.
Community treatment and prevention

Eradication of cysticercosis should be possible by removing it from either pig or human hosts, or both. Reform of animal husbandry techniques, meat inspection procedures, and adequate cooking of pork are difficult approaches and of limited relevance in developing countries, where pigs are free roaming, or raised by subsistence farmers who cannot afford enclosed pens or proper animal feed, and meat is sold off outside the abattoir system. Vaccination of pigs and immunotherapy have been proposed as measures to break the parasite life cycle. Partial protection against porcine cysticercosis has been demonstrated. ${ }^{51-53}$ Taenicidal eradication in humans may have adverse effects in people with occult neurocysticercosis, who may become ill when cysticerci die. ${ }^{54}$ Studies of mass treatment with praziquantel may produce early benefit, but longer term evaluation shows no lasting impact.

\section{Management of epilepsy in the developing world}

EPIDEMIOLOGY

Epilepsy affects 5-10/1000 population throughout the world, with $75 \%$ of cases arising in childhood. ${ }^{1}$ Neurocysticercosis is a major cause in developing countries, ${ }^{45}{ }^{12}$ but the relative contribution to all incident cases is unknown. Malaria and other parasites are associated with epilepsy. ${ }^{55-57}$ Studies in Ecuador, Tanzania, Nigeria, and Pakistan have consistently found higher prevalence of epilepsy in rural than in urban areas using identical methodologies, further suggesting that infectious diseases may be an important aetiological factor for epilepsy in the developing world. ${ }^{58-61}$ New epidemiological associations have been suggested between low body mass index, previous adverse reproductive experience, recent infective illness, and risk of epilepsy in children. ${ }^{62-64}$ These interesting associations merit further study in developing countries, as they may disclose new mechanisms of risk and risk interaction for focal brain damage in childhood.

IMPACT

Everyday conditions for most of the world's poor are radically different from the experience of the western hospital outpatient. This context must be appreciated when considering the impact of epilepsy. The primary concern of families is often subsistence, and this shapes their attitudes to health and their contact with health services. Poor female literacy, often associated with underdevelopment, is an important influence on child health, whereas cultural and religious beliefs may also impinge on health related attitudes and practices.

Few studies have measured the impact of epilepsy on family life in developing countries. One population study of childhood epilepsy in rural India has shown that epilepsy has pervasive effects on social adjustment, which probably do not spontaneously resolve..$^{65}$ One third of children had motor or cognitive impairment and one third had intractable seizures. Only $50 \%$ went to school compared with over $95 \%$ 
of their peers. Difficulties of parental adjustment, reported in north American studies, ${ }^{66}$ were even more prominent in rural India, with marked maternal depression. ${ }^{65} \mathrm{~A}$ strong social support network helped with parental adjustment. ${ }^{65}$

The economic impact of epilepsy has not been prospectively studied, but estimates from rural India suggest that the social and financial cost of hospital attendance is a major disincentive to continued treatment. ${ }^{65}$ India (until recently) and other countries also have laws that discriminate against people with epilepsy-for example, with regard to marriage, employment, and insurance. ${ }^{67}$ For practical purposes epilepsy must therefore be considered a disability and interventions should consider all aspects.

\section{INTERVENTION}

The type of intervention required, as suggested by studies of impact, and the necessary resources for service delivery are not widely available in the developing world. The "treatment gap" refers to the proportion of people with epilepsy untreated with antiepileptic drugs on any given day. The estimates in developing countries range from $80 \%-94 \% .^{3}$ The causes of the treatment gap have not yet been systematically studied but they must be multiple, overlapping, and varying between countries. They may be considered at infrastructural, health sector, and community levels. For example, some countries may have established health systems but lack finances or reliable drug supplies. In some communities, there may be preferred alternatives to antiepileptic drug treatment. Generalisation is difficult. There are several themes that should be considered in the design of services in developing countries: ascertainment, disability, intractability, sustainability, equity, community involvement, and financing. ${ }^{68}$

The first issue is that of ascertainment. In many communities, epilepsy is a stigmatising disorder. House to house surveys are thought to be an epidemiologically sensitive method of finding cases. However, in our experience, they may cause unnecessary distress to families and the community. They are an expensive method, and sensitivity can be as low as $60 \%$ because of concealment. ${ }^{69}$ The use of key informants, or leading members of the community, to identify people with epilepsy, has several distinct advantages. These include the opportunity to explain the aims of the service, finding out local priorities, and working with the community to overcome social barriers to integration. Strong community involvement is also a key element to ensuring sustainability.

A third of people with epilepsy have physical or cognitive difficulties. Whether or not seizures can be controlled, people with epilepsy need to resume as normal a life as possible. This requires a holistic assessment and formulation of an action plan with the community. This is especially important for children's development. This kind of intervention can only be planned and executed at a local level. The disability aspect of epilepsy is perhaps the most important and neglected aspect of intervention.

An intractability rate of one third should be expected. Ideally, primary facilities need to have links with specialists for advice and referral. In some areas epilepsy surgery programmes may be offered, but opportunities for postoperative rehabilitation and reintegration should be major considerations before planning surgery.

The treatment and rehabilitation of people with epilepsy is a long term matter, and so it is essential that services, once started, continue in a predictable way. Sustainability of health programmes is associated with strong community ownership, political will, and stable financing. Services should be cheap to set up and run, and this can be achieved at marginal cost by integrating epilepsy services alongside existing medical or welfare programmes, rather than starting a new vertical programme.

It is well known that those most in need of health provision are often least able to gain access to it. A central tenet of primary health services is equity. Our studies of dropout showed that a significant proportion could not afford time away from home or work to attend clinics, despite wanting to continue follow up. ${ }^{65 a}$ Hospital based services (in India) have very high attrition rates and so are ill equipped to provide necessary long term monitoring and treatment. ${ }^{65}$ Flexibility in delivering services is therefore important to prevent attrition, ideally to the door if necessary. Services can be extended through community mental health and community based rehabilitation agencies, in both state and non-governmental sectors. ${ }^{70}$ A community based approach can fulfil the essential aim of rehabilitation through effective case holding, thus minimising dropout. Low cost is also a major motive for reattendance, and therefore precludes expensive investigations or new generation drugs at first contact. Community based services still need to be affordable by their users.

Expertise in dealing with epilepsy has to be devolved to local health personnel to attain wide coverage. In many countries, epilepsy is not covered in the medical undergraduate curriculum, and most general practitioners are not familiar with routine management. Training is required at all levels, and systems of monitoring and evaluation designed for local use.

The use of phenobarbital as first line agent remains very controversial among clinicians. Although the most widely available antiepileptic drug in the world, and effective against many seizure types, its use has been discouraged in some quarters because of concern about excessive side effects in children. However, a recent clinical trial has established that phenobarbital is indeed acceptable as a first line antiepileptic drug for children in India, ${ }^{71}$ and this is supported by a population study which refutes the notion that it is often used as a drug of misuse or for suicides among adults. ${ }^{72}$ In many parts of the world, the supply of antiepileptic drug is unreliable, and the choice may be between phenobarbital or nothing. ${ }^{73}$ 
Epilepsy control programmes will need detailed resource planning. Training, drugs, and infrastructural development will need costing. The non-governmental and private sectors play an increasing role in the provision of primary services in India. Physical standards are often superior, and acceptance by mothers and children is high. Many non-governmental organisations are oriented towards community development and use village health workers. A collaboration of sectors is necessary in countries like India where the public system alone cannot achieve wide coverage.

The unmet needs of people with epilepsy in developing countries are obviously more complex than providing treatment with drugs. Appropriate interventions must consider the medical, developmental, and psychosocial needs of people with epilepsy, as well as being financially, geographically, and culturally accessible. Legal barriers to social advancement also need to be removed. Research is urgently needed on the population risk factors for epilepsy in developing countries, and action has to be coordinated against known preventable causes.

\section{Conclusions}

Relatively little is known about the epidemiology of neurocysticercosis, and cysticidal treatments have been advocated without clear evidence for overall benefit in humans. Diagnosis may be difficult even with neuroimaging and serological facilities. Neurocysticercosis is a major cause of acquired epilepsy in Latin American countries. The economic burden of both are not well quantified. Community prevention and eradication of neurocysticercosis depends on general infrastructural development. Most people with epilepsy in developing countries do not have access to appropriate management. The major challenge for the future will be to develop primary level services that provide appropriate interventions for these communities.

1 Sander JW, Shorvon SD. Epidemiology of the epilepsies. $\mathcal{F}$ Neurol Neurosurg Psychiatry 1996;61:433-43.

Bertelotte JM. Epilepsy as a public health problem. Trop Geogr Med 1994;46:28-30.

3 Shorvon SD, Farmer PJ. Epilepsy in developing countries: a review of epidemiological, sociocultural and treatment aspects. Epilepsia 1988;29(suppl 1):36-54.

4 Garcia HH, Gilman R, Martinez M, et al. Cysticercosis as a major cause of epilepsy in Peru. Lancet 1993;341:197-200.

5 Medina M, Rosas E, Rubio F, et al. Neurocysticercosis as the main cause of late-onset epilepsy in Mexico. Arch Intern Med 1990;150:325-7.

6 White AC. Neurocysticercosis: a major cause of neurological disease worldwide. Clin Infect Dis 1997;24:101-15.

7 Carpio A, Escobar A, Hauser W. Epilepsy and cysticercosis: a critical review. Epilepsia 1998;39:1025-40.

8 Craig PS, Rogan MT, Allan JC. Detection, screening and community epidemiology of taeniid cestode zoonoses: cystic echinococcosis, alveolar echinococcosis and neurocysticercosis. Adv Parasitol 1996;38:170-250.

9 Del Brutto OH. Neurocysticercosis. Curr Opin Neurol 1997; 10:268-72.

10 Powell SY, Proctor AJ, Wilmot B, et al. Cysticercosis and epilepsy in Africans: a clinical and neurological study. Ann Trop Med Parasitol 1966;60:142-58.

11 Dumas M, Grunitzky E, Deniau M, et al. Epidemiological study of neurocysticercosis in northern Togo (west Africa). Acta Leidensia 1989;57:191-6.

12 Garcia-Noval J, Allan JC, Fletes C, et al. Epidemiology of Taenia solium taeniasis and cysticercosis in two rural GuaTaenia solium taeniasis and cysticercosis in two rural Gua-

13 Sanchez AL, Gomez O, Allebeck P, et al. Epidemiological study of Taenia solium infections in a rural village in Honduras. Ann Trop Med Parasitol 1997;91:163-71.
14 Sarti E, Schantz PM, Plancarte A, et al. Prevalence and risk factors for Taenia solium taeniasis and cysticercosis in
humans and pigs in a village in Morelos, Mexico. Am $\mathcal{F}$ Trop Med Hyg 1992;46:677-85.

15 Cao W, van der Ploeg CPB, Xu J, et al. Risk factor for human cysticercosis morbidity: a population based casecontrol study. Epidemiol Infect 1997;119:231-5.

16 Goodman K, Ballagh SA, Carpio A. Case control study of seropositivity for cysticercosis in Cuenca, Ecuador. Am $\mathcal{F}$ Trop Med Hyg 1999;60:70-4.

17 Schantz PM. Cysticercosis in non-endemic countries: the example of the United States. In: Garcia HH, Martinez SM, eds. Teniasis/cisticercosis por T solium. Lima: Editorial Universo, 1996.

18 Roberts T, Murrell KD, Marks S. Economic losses caused by food borne parasitic diseases. Parasitology Today 1994;11:419-23.

19 Dixon HBF, Lipscomb FM. Cysticercosis: an analysis and follow-up of 450 cases. Privy Council Medical Research Special Report Series 1961;229:1-58.

20 MacArthur WP. Cysticercosis as seen in the British Army, with special reference to the production of epilepsy. Transactions of the Royal Society of Tropical Medicine and Hygiene 1934;27:343-63.

21 Rolfs A, Muhlschlegel F, Jansen-Rosseck R, et al. Clinical and immunologic follow-up study of patients with neurocysticercosis after treatment with praziquantel. Neurology 1995;45:532-8.

22 Shambesh MK, Craig PS, Wen $\mathrm{H}$, et al. IgG1 and IgG4 serum antibody responses in asymptomatic and clinically expressed cystic echinococcosis patients. Acta Tropica 1997;64:53-63.

23 Evans CWE, Garcia HH, Hartnell A, et al. Elevated concentrations of eotaxin and interleukin- 5 in human neurocysticercosis. Infect Immun 1998;66:4522-5.

24 Kramer LD, Locke GE, Byrd SE, et al. Cerebral cysticercosis: documentation of natural history with CT. Radiology 1989;171:459-62.

25 Thussu A, Sehgal S, Sharma M, et al. Comparison of cellular responses in single and multiple lesion neurocysticercosis. Ann Trop Med Parasitol 1997;91:627-32.

26 Wadia NH. Neurocysticercosis. In: Shakir RA, Newman PK, Poser CM, eds. Tropical neurology. London: Saunders, 1996:247-73

27 Jain S, Padma MV, Kanga U, et al. Human leukocyte antigen studies in Indian probands with seizures associated with single small enhancing computed tomography lesions and seizure types in their family members. Fournal of Epilepsy seizure types in

28 Hill AVS. Genetics of infectious disease resistance. Curr Opin Genet Dev 1996;6:348-53.

29 Almari A, Batchelor JR. HLA and hepatitis B infection. Lancet 1994;344:1194-5.

30 Fernandez-Reyes D, Craig A, Kyes SA. A high frequency African coding polymorphism in the $\mathrm{N}$-terminal domain of ICAM-1 predisposing to cerebral malaria in Kenya. Hum Mol Genet 1997;6:1357-60.

31 Rajshekar V, Chacko G, Haran RP, et al. Clinicoradiological and pathological correlations in patients with solitary Cysticercus granuloma and epilepsy: focus on presence of the icercus granuloma and epilepsy: focus on presence of the parasite and $695 ; 59: 284-6$.

32 Del Brutto OH, Santibanez R, Noboa CA, et al. Epilepsy due to neurocysticercosis: analysis of 203 patients. Neurology 1992;42:389-92.

33 Kalra V, Sethi A. Childhood neurocysticercosis. Epidemiology, diagnosis and course. Acta Paediatr fpn 1992;34:36570 .

34 Monteiro L, Nunes B, Mendonca D, et al. Spectrum of epilepsy in neurocysticercosis: a long term follow-up of 143 patients. Acta Neurol Scand 1995;92:33-40.

35 Singh G. Neurocysticercosis in South-Central America and the Indian subcontinent: a comparative evaluation. Arq Neuropsiquiatr 1997;55:349-56.

36 Rajshekar V. Etiology and management of single small CT lesions in patients with seizures: understanding a controversy. Acta Neurol Scand 1991;84:465-70.

37 Wadia RS, Makhale CN, Kelkar AN, et al. Focal epilepsy in India with special reference to lesions showing ring or disc like enhancement on contrast computed tomography. $\mathcal{F}$ Neurol Neurosurg Psychiatry 1987;50:1298-301.

38 Rajshekar V, Haran RP, Prakash S, et al. Differentiating solitary small cysticercus granulomas and tuberculomas in patients with epilepsy. $\mathcal{F}$ Neurosurg 1993;78:402-7.

39 Rosenfeld EA, Byrd SE, Shulman ST. Neurocysticercosis among children in Chicago. Clin Infect Dis 1996;23:262-8.

40 Ramos-Kuri M, Montoya RM, Padilla A, et al. Immunodiagnosis of neurocysticercosis. Disappointing performance of serology (ELISA) in an unbiased sample of neurological patients. Arch Neurol 1992;49:633-6.

41 Schantz PM, Sarti E, Plancarte A, et al. Community-based epidemiological investigations of cysticercosis due to Taenia solium: comparison of serological screening tests and clinical findings in two populations in Mexico. Clin Infect Dis 1994;18:879-5.

42 Tsang V, Brand A, Boyer A. An enzyme linked immunoelectrotransfer blot assay by glycoprotein antigens for diagnosing human cysticercosis (Taenia solium). F Infect Dis 1989; 159:50-59.

43 Rajshekhar V, Wilson M, Schantz PM. Cysticercus immunoblot assay in Indian patients with single, small enhancing CT lesions. F Neurol Neurosurg Psychiatry 1991;54:561. 
44 Garcia HH, Harrison LJS, Parkhouse RME, et al. A specific antigen-detection ELISA for the diagnosis of human
neurocysticercosis. Trans Med Soc Trop Med Hyg 1998;92: neurocys

45 Del Brutto OH, Wadia NH, Dumas M, et al. Proposal of diagnostic criteria for human cysticercosis and neurocysticercosis. F Neurol Sci 1996;142:1-6.

46 Carpio A. Diagnostic criteria for human cysticercosis. $\mathcal{F}$ Neurol Sci 1999;161:185-8.

47 Mitchell WG, Crawford TO. Intraparenchymal cerebral cysticercosis in children: diagnosis and treatment. Pediatrics 1988;82:76-82

48 Wadia N, Desai S, Bhatt M. Disseminated cysticercosis. New observations, including CT scan findings and experience with treatment with praziquantel. Brain 1988;111: 597-614.

49 Salinas R, Prasad K. Curative drug therapy in human neurocysticercosis. In: Garner P, Gelband $\mathrm{H}$, Olliaro $\mathrm{P}$, et al, eds. Tropical disease module of the Cochrane database of systematic reviews (updated 12 June 1996). Oxford: The systematic reviews (updated

50 Baranwal AK, Singhi PD, Khandelwal N, et al. Albendazole therapy in children with focal seizures and single small enhancing computerised tomographic lesions: a randomized, placebo-controlled, double blind trial. Pediatr Infect Dis ₹ 1998;17:696-700.

51 Molinari JL, Soto R, Tato P, et al. Immunization against porcine cysticercosis in an endemic area in Mexico: a field and laboratory study. Am F Trop Med Hyg 1993;49:502-12.

52 Pathak KML, Gaur SMS. Immunisation of pigs with culture antigens of T solium. Vet Parasitol 1980;34:353-6.

53 Evans CA, Gonzalez AE, Gilman RH, et al. Immunotherapy for porcine cysticercosis: implications for prevention of human disease Cysticercosis Working Group in Peru. Am f Trop Med Hyg 1997;56:33-7.

54 Flisser A, Madrazo I, Plancarte A, et al. Neurological symptoms in occult neurocysticercosis after single taeniacidal dose of praziquantel. Lancet 1989;342:748.

55 Kaiser C, Kipp W, Asaba G, et al. The prevalence of epilepsy follows the distribution of onchocerciasis in a West follows the distribution of onchocerciasis in a west

56 Waruiru CM, Newton CRJC, Forster D, et al. Epileptic seizures and malaria in Kenyan children. Trans Med Soc Trop Med Hyg 1996;90:152-5.

57 Senanayake N, Roman GC. Epidemiology of epilepsy in developing countries. Bull World Health Organ 1993;71 247-58.

58 Aziz H, Ali SM, Frances P, et al. Epilepsy in Pakistan: a population-based epidemiological study. Epilepsia 1993;35: 950-8.
59 Placencia M, Shorvon SD, Paredes V, et al. Epileptic seizures in Andean region of Ecuador: incidence and seizures in Andean region of Ecuador: incidence and

60 Rwiza HT, Kilonzo GP, Haule J, et al. Prevalence and incidence of epilepsy in Ulanga, a rural Tanzanian district: a community-based study. Epilepsia 1992;33:1051-6.

61 Osuntokun BO, Adeuja AOG, Nottidge VA, et al. Prevalence of the epilepsies in Nigerian Africans: a communitybased study. Epilepsia 1987;28:272-9.

62 Hackett RJ, Hackett L, Bhakta P. The prevalence and associated factors of epilepsy in children in Calicut district, Kerala, India. Acta Paediatrica 1997;86:1257-60.

63 Kuenneth CA, Boyle C, Murphy CC, et al. Reproductive risk factors for epilepsy among 10 year old children in metropolitan Atlanta. Paediatr Perinat Epidemiol 1996;10:186ropolit.

$64 \mathrm{Pal} \mathrm{DK}$. Methodological considerations in assessing epilepsy risk factors in an epidemiological study in India. Neurology 1999;53:1-6.

$65 \mathrm{Pal}$ DK. Control of childhood epilepsy in rural India. Evaluation of a community based intervention [ $\mathrm{PhD}$ thesis]. London: University of London, 1998.

65a Pal DK, Das T, Sengupta S. Case-control and qualitative study of attrition in a community epilepsy programme in rural India, Seizure 2000; (in press)

66 Austin JK, McDermott N. Parental attitude and coping behaviours in families of children with epilepsy. 7 Neurosci burs 1988;20:174-9.

67 Mani KS, Hedge NS. Epilepsy and law: position in India. Advances in epileptology. Vol 17. New York: Raven Press, 1989

68 Pal DK, Nandy S, Sander JWAS. Towards a coherent public health analysis for epilepsy. Lancet 1999;353:1817-8.

$69 \mathrm{Pal} \mathrm{DK}$, Das T, Sengupta S. Comparison of key informant and survey methods for ascertainment of childhood epilepsy in West Bengal, India. Int $\mathcal{F}$ Epidemiol 1998;27: $672-6$.

70 World Health Organisation. Community control of epilepsy: report on an informal consultation for the development of a report on an informal consultation for the
strategy and protocol. Geneva: WHO, 1985.

71 Pal DK, Das T, Chaudhury G, et al. Randomised controlled trial to assess acceptability of phenobarbital for childhood epilepsy in rural India. Lancet 1998;351:19-23.

72 Mani KS. Global campaign against epilepsy. Agenda for IEA/IES. Neurology India 1998;46:1-4.

73 III and IV Commissions on Antiepileptic Drugs of the International League Against Epilepsy. Availability and distribution of antiepileptic drugs in developing countries. Epilepsia 1985;26:117-21. 